\title{
The anesthesiologist and end-of-life care
}

\author{
Sebastiano Mercadante ${ }^{\mathrm{a}, \mathrm{b}}$ and Antonello Giarratano ${ }^{\mathrm{b}}$
}

\begin{abstract}
Purpose of review
Anesthesiologists may face problematic situations when patients are close to death, in which clinical problems, decision-making processes, and ethical issues are often interconnected and dependent on each of them. The aim of this review is to assess the recent literature regarding the anesthesiological role for advanced cancer patients.
\end{abstract}

\section{Recent findings}

Palliative sedation in the dying patients, end-of-life problems in the ICU, and pain control in advanced cancer patients have been the subject of recent research. All these issues have shown that anesthesiologist would be expert in the field of pain and symptom control at the end of life. End-of-life care problems are common in ICU, and a decision-making process requires knowledge and management of patients' wishes, past and projected future quality of life, severity and prognosis of illness, patients' age, regarding withholding and withdrawing of futile treatments in anticipation of death, or relieving symptoms close to death.

\section{Summary \\ Anesthesiologists should be competent in all aspects of terminal care, including the practical and ethical aspects of withdrawing different modalities of life-sustaining treatment and the use of sedatives, analgesics, and nonpharmacologic approaches to easing the suffering of the dying process. More research is needed to provide models which should be spread in the scientific community to afford this difficult task.}

\section{Keywords}

cancer pain, end of life, palliative care, symptom control, terminal sedation

\section{INTRODUCTION}

Anesthesiologists may face problematic situations, in which clinical problems, decision-making processes, and ethical issues are often interconnected and dependent on each of them. Although it would be desirable that anesthesiologists would be expert in the field of pain and symptom control at the end of life, work load is often an insurmountable obstacle to a direct and continuous care of advanced cancer patients. In most cases, consultations are performed to provide advice for difficult cases or interventional procedures. Moreover, end-of-life care problems are common in the ICU, and decisionmaking process requires knowledge and management of patients' wishes, past and projected future quality of life, severity and prognosis of illness, patients' age, regarding withholding and withdrawing of futile treatments in anticipation of death, or relieving symptoms close to death. The aim of this review is to assess the recent literature regarding the anesthesiological role for advanced cancer patients. Very few studies assessed this topic, particularly in the last year, underlining the need of much research in this field.

\section{DIFFICULT TASKS IN ADVANCED CANCER PATIENTS}

An example of a complex decision-making process is recurrent malignant bowel obstruction. Bowel obstruction because of recurrent cancer is commonly associated with poor long-term survival. Surgical attempts to relieve malignant obstruction have a significant morbidity and mortality, and variable success in resolving symptoms. The presence of carcinomatosis strongly influences surgical prognosis. Surgical intervention in patients with peritoneal carcinomatosis produces rather short-term success with significant mortality and morbidity rates related to the procedure, ranging

\footnotetext{
${ }^{a}$ Anesthesia \& Intensive Care and Pain Relief \& Supportive Care Unit, La Maddalena Cancer Center and ${ }^{b}$ Chair of Anesthesiology, University of Palermo, Italy

Correspondence to Dr Sebastiano Mercadante, Anesthesia \& Intensive Care and Pain Relief \& Supportive Care Unit, La Maddalena Cancer Center, Palermo, Italy. Tel: +39 916806521; fax: +39 916806110; e-mail: terapiadeldolore@lamaddalenanet.it/03sebelle@gmail.com
}

Curr Opin Anesthesiol 2012, 25:371-375

DOI:10.1097/ACO.0b013e3283530e7d 


\section{KEY POINTS}

- ICU clinicians should be competent in all aspects of the end-of-life care.

- Integration of palliative care experts into the operation of ICUs may be of benefit to patients, families, and critical care clinicians.

- Communication issues with the relatives are essential, as an appropriate relationship improves patient care and family outcomes in the last days of life, allowing us to share the same objectives and expectations.

between 14-29 and 37-45\%, respectively. Apart from obstruction, peritoneal carcinomatosis may cause motility problems because of intestinal paralysis secondary to extensive tumor involvement of the intestinal mesentery and plexuses, which is not cured by surgical procedures. Survival rates of medically versus surgically treated patients have been found to be not significantly different. Anesthesiologists should be aware that in any case, expected survival time is not necessarily an absolute factor for decision-making, as deterioration of patients' condition in the postoperative period, suffering from complications and hospitalization, in other terms, quality of life, should also be taken into account. Postoperative complications include wound infections and dehiscence, sepsis, enterocutaneous fistula, further obstruction, peritoneal abscess, anastomosis dehiscence, gastrointestinal bleeding, pulmonary embolism, and deep venous thrombosis. Successful palliation in terms of duration of maintenance of intestinal transit and gastrointestinal symptom control is the most important outcome. These factors are difficult to explore in comparison with the standard key-points of prognosis, like mortality and morbidity rates, while quality-of-life measurements remain difficult to apply in this context. Prognostic criteria are difficult to apply in a condition that is highly variable individually, whose assessment is not uniformly recognized, approaches as well as diagnostic and therapeutic choices are dependent on the setting, definition of the outcome is rarely afforded, and available data on survival prognosis of the illness cannot be drawn for practical scoring. The multidisciplinary approach, including the opinion of the anesthesiologist and the surgeon, as well as an expert in palliative medicine, is rarely performed, and despite the possible poor prognosis, surgery is often performed, because it is considered inevitably as an imperative act (otherwise the patient will die). Surgery should be justified on the basis of more benefit rather than burden to patients, based on the prognostic factors available, and consent to surgery should include discussion of risks, complications, as well as medical alternatives, effective in relieving the symptoms [1"'].

Patients who are considered inoperable after a careful consideration of the individual clinical condition should be managed in a different context addressing the quality-of-life issues, rather than survival. The pharmacological management of malignant bowel obstruction in inoperable malignant bowel obstruction focuses on the relief of nausea and vomiting, pain, and other possible distressing symptoms. It principally consists of an association of antiemetics, antisecretory drugs, and analgesics. In bowel obstruction, a combination of corticosteroids and antisecretory agents is commonly used. Specifically, an anticholinergic drug or octreotide may reduce, by different mechanisms, pain complaints due to abdominal distension, other than decreasing gastrointestinal secretion [1"'].

\section{TERMINAL SEDATION}

Although sedation for symptom relief in terminally ill patients has been the focus of recent medical studies, the interpretation of research findings is difficult because of confusing terminology. Palliative sedation therapy should be considered as the use of sedative medications to relieve intolerable and refractory distress by the reduction in patient's consciousness. This should not be confounded with a slow euthanasia when clinicians sedate patients approaching the end of life with the primary goal of hastening the patient's death. Rather, there is no proof that sedation is associated with a shorter survival [2"]; thus, the concept of the double effect, which should justify the intent of the good effect (relieving suffering), should not be the determinant. This practice is universally used in the ICU for short periods, but not considered as definitive as in this case. In palliative setting, this practice is common, ranging in about $10-50 \%$ of dying patients depending on the setting. Most centers use a midazolam-based regimen because of the drug's short half-life, ease of intravenous or subcutaneous administration, and good efficacy. Opioids should not be used for the primary purpose of sedation, but rather should be continued for analgesic purposes or doses tailored to control dyspnea [3"].

\section{END-OF-LIFE PROBLEMS IN THE INTENSIVE CARE UNIT}

Dying has changed as a process over the last century in terms of causes of death, costs, communication of 
the prognosis, and needs of the patient's family. Nearly half of Americans who die in hospitals spend time in the ICU in the last 3 days of life [4"]. The consequent possibility of manipulating the dying process has generated many problems, which range from the necessity of rationing scarce and expensive resources to the definition of optimal care for dying people and the necessity of redefining death itself. As a consequence, actions should be accompanied by careful bioethical reflection [ $\left.5^{\circ}\right]$. The ICU represents a hospital setting in which death and discussion about the end-of-life care are common, yet these conversations are often difficult. Minority patients who die in the ICU are less likely to formalize advance directives, and surviving family members report lower satisfaction with the provision of information and sensitivity to their cultural traditions at the end of life [4"]. Such difficulties arise, in part, because a family may be facing an unexpected poor prognosis associated with an acute illness or exacerbation and, in part, because the ICU orientation is one way of saving lives. Understanding and improving communication about the end-of-life care between clinicians and families in the ICU is an important focus for improving the quality of care in the ICU. Unfortunately, a considerable variation in the apparent willingness of consultants to make endof-life decisions has been reported, emphasizing the subjective nature of these decisions [6]. High-quality care for the ICU patients and their families includes palliative care. Quality indicators of palliative care processes, such as identification of medical decisionmaker, advance directive and resuscitation preference, distributing family information leaflet, assessing and managing pain, offering social work and spiritual support, and conducting an interdisciplinary family meeting, have been measured in the ICU. The performance was inconsistent and infrequent, other than pain assessment and management [7"'].

ICU clinicians should be competent in all aspects of this care, including the practical and ethical aspects of withdrawing different modalities of life-sustaining treatment and the use of sedatives, analgesics, and nonpharmacologic approaches to easing the suffering of the dying process. Recommendations based on ethical and legal principles, rather than deriving from evidence, have been developed to improve the care for the ICU patients during the dying process [8]. In a study investigating influencing decisions to withdraw life support, rather than age or the severity of the illness and organ dysfunction, the strongest determinants of the withdrawal of ventilation in critically ill patients were the physician's perception that the patient preferred not to use life support, the physician's prediction of a low likelihood of survival in the ICU and a high likelihood of poor cognitive function, and the use of inotropes or vasopressors [9]. A total of $55 \%$ of ICU deaths were the result of treatment withdrawal. Overwhelmingly, treatment failure or futility was the reason cited for withdrawal. There were no cases of conflict between the medical team and the patient's family. Consultation with the ICU colleagues was rated as the most helpful factor in decision-making [10]. In a study of policies of withholding and withdrawal of life-sustaining treatment in critically ill patients on cardiac ICUs, cranial computed tomography with poor prognosis, multiorgan failure, and failure of assist device therapy were the most frequently cited medical reasons for withholding or withdrawal of life-sustaining treatments, while poor expected quality of life, the patient's willingness to limit medical care, and the family's choice were the top ethical reasons [11].

The knowledge of the distinctions between withholding and withdrawing treatments, between actions of killing and allowing to die, and between consequences that are intended and those that are merely foreseen is of paramount importance. Communication issues with the relatives are essential, as an appropriate relationship improves patient care and family outcomes in the last days of life, allowing to share the same objectives and expectations. Respect for patient autonomy and the intention to honor decisions to decline unwanted treatments should be conveyed to the family. If a conflict cannot be resolved, an ethics consultation may be helpful [12]. The effectiveness of discussions that take place between clinicians and family members becomes a crucial marker of high-quality intensive care [13].

Another aspect linked to the end-of-life care in the ICU includes the ability for notifying families of a patient's death and compassionate approaches for discussing the options for organ donation. Comprehensive bereavement programs to support both families and the needs of the clinical staff should be included in the educational curricula for improving the end-of-life care in the ICU. An audit study demonstrated the feasibility of developing a quality improvement tool [14] for the end-of-life decision-making in the intensive care setting. The potential for strained communications is mitigated, if clinicians provide timely clinical and prognostic information and support family with continuous psychological support. Effective communication includes sharing the burden of decision-making with family members. This shift from individual responsibility to patient-focused 
consensus often permits the family to understand that intensive caring may involve letting go of life-sustaining interventions [15]. Family members' informal roles are part of families' decision-making processes, as they determine the complicated family dynamics within the family system and between the family and heath care domains. Identification and description of these informal roles of family members can help the physician recognize and understand the functions of these roles in families' decision-making at the end of life and guide the development of strategies to support and facilitate increased effectiveness of family discussions and decision-making processes [16"]. Other demographic variables should be considered among the visitors at the ICU. For example, professionals should pay special attention to the needs of younger visitors to the ICU, women, and those with a low level of education, as they may require more support, comfort, and proximity [17].

Integration of palliative care experts into the operation of ICUs may be of benefit to patients, families, and critical care clinicians. A palliative care team dramatically changed the way to treat dying patients in the ICU in comparison with patients who died without a palliative care consultation [4"]. Education on the death process was provided to $85 \%$ of families by the project team. A total of $29 \%$ of patients were disconnected from mechanical ventilators following consultation with the Palliative Care Service: $15.9 \%$ of patients discontinued the use of inotropic support, 15.3\% stopped artificial nutrition, $6.4 \%$ stopped dialysis, and $2.5 \%$ discontinued artificial hydration. Recommendations on pain management were made for $51 \%$ of the project's patients and symptom management for $52 \%$ of patients. The project was associated with an increase in the rate of the formalization of advance directives. A total of $33 \%$ of the patients who received palliative care consultations had 'do not resuscitate' orders in place prior to consultation and $83.4 \%$ had 'do not resuscitate' orders after the intervention. The project team referred 80 (51\%) of the project patients to hospice and 55 (35\%) patients were enrolled on hospice, primarily at the medical center. The mean time from admission to palliative care consultation at the project site was 2.8 versus 15.5 days at the other campus without a palliative care team. Median survival times from admission to the medical center were not significantly different. Median charges for the use of opioid medications were higher for the intervention group but lower for use of laboratory and radiology tests. This study suggests that such models may be associated with improved quality of life, higher rates of formalization of advanced directives and utilization of hospices, as well as lower use of certain nonbeneficial life-prolonging treatments for critically ill patients who are at the end of life.

\section{PAIN CONTROL IN ADVANCED CANCER PATIENTS}

When symptoms are severe, anesthesia-pain medicine and palliative medicine can effectively treat the symptoms. In a small study, the palliative medicine group resulted in being more effective in symptom management and improving the quality of life, in comparison with the anesthesia-pain medicine group, the differences in pain relief being less relevant [18"]. This small study suggests that anesthesiologists require a more comprehensive knowledge about the problems of advanced cancer patients, as symptom management could be suboptimal. On the other hand, interventional procedures are performed by skilled people, commonly anesthesiologists. Although the need of these techniques has been decreasing in the last years, because of a better knowledge about the use of opioids and a better drug availability, this does not mean that the selected population would be proposed for an interventional procedure. Only $25 \%$ of anesthesiologists' job plans had time allocated for palliative medicine referrals [19]. A small number of patients may still fail to obtain adequate analgesia despite large systemic opioid doses or they may suffer from uncontrollable side-effects such as nausea, vomiting, or oversedation. Nerve blocks, neuroaxial infusions, and radiofrequency ablations are viable options to enhance the pain control in cancer patients. Minimally invasive vertebral procedures have been proposed in recent years to provide pain relief. However, risks may be of concern, particularly in the advanced stage of disease. For example, the interruption of sympathetic nervous system to treat pain is the least innocuous block in terms of complications. However, it has been shown that a neurolytic sympathetic plexus block is more efficacious when considered earlier in the disease [20]. Neural blockade is performed in a minority of patients who receive specialized palliative care. Common procedures included epidurals, neurolytic plexus block, and intrathecal nerve block with phenol [21].

In the 1990s, spinal analgesia has been described as a useful means to control pain in advanced cancer patients. Despite a lack of clinical evidence, spinal analgesia with a combination of opioids, principally morphine, and local anesthetics may allow us to achieve analgesia in patients who had been intensively treated unsuccessfully with different trials of opioids. Some adjuvant drugs such as clonidine, ketamine, betamethasone, meperidine, 
and ziconotide may be promising agents, but several problems have to be solved before they can be used in daily practice. In complex pain situations, spinal analgesia should not be negated to cancer patients, and oncologists should address this group of patients to anesthesiologists [22].

\section{CONCLUSION}

Anesthesiologists should be competent in all aspects of palliative care and the end of life, including the practical and ethical aspects of withdrawing different modalities of life-sustaining treatment and the use of sedatives, analgesics, and nonpharmacologic approaches to easing the suffering of the dying process. Integration of palliative care experts into the operation of ICUs may be of benefit to patients, families, and critical care clinicians.

\section{Acknowledgements}

None.

\section{Conflicts of interest}

There are no conflicts of interest.

No grants and economical support were received for this study.

\section{REFERENCES AND RECOMMENDED \\ READING}

Papers of particular interest, published within the annual period of review, have been highlighted as:

- of special interest

-n of outstanding interest

Additional references related to this topic can also be found in the Current

World Literature section in this issue (pp. 395-396).

1. Mercadante S, Porzio G. Octreotide for malignant bowel obstruction: twenty nears after. Crit Rev Oncol Hematol 2012. [Epub ahead of print]

This study describes the historical process of octreotide, which is an important component of the medical treatment of inoperable bowel obstruction in patients who had been considered for years to be candidates to surgery despite the bad prognosis.

2. Juth N, Lindblad A, Lynöe N, et al. European Association for Palliative Care

- (EAPC) framework for palliative sedation: an ethical discussion. BMC Palliat Care 2010; 9:20.

Ethical issues regarding palliative sedation discussed by the experts in palliative care.
3. Olsen M, Swetz K, Mueller P. Ethical decision making with end-of-life care: - palliative sedation and withholding or withdrawing life-sustaining treatments. Mayo Clin Proc 2010; 85:949-954.

A study presenting the most important issue regarding the decision-making process at the end of life.

4. O'Mahony S, McHenry J, Blank AE, et al. Preliminary report of the integration

- of a palliative care team into an intensive care unit. Palliat Med 2010; 24:154165.

A study assessing the need of integration between palliative care and ICU.

5. Zamperetti N, Piccinni P. End of life in the intensive care unit. Minerva

- Anestesiol 2010; 76:541-547.

Presentation of end-of-life problems in the ICU.

6. Poulton B, Ridley S, Mackenzie-Ross R, Rizvi S. Variation in end-of-life decision making between critical care consultants. Anaesthesia 2005; 60: $1101-1105$.

7. Penrod J, Provonost P, Livote E, et al. Meeting standards of high-

- quality intensive care unit palliative care: clinical performance and predictors. Crit Care Med 2012. [Epub ahead of print]

A study assessing the need of high-quality standards in ICU, including the experience of a palliative care team.

8. Truog RD. End-of-life decision-making in the United States. Eur J Anaesthesiol Suppl 2008; 42:43-50.

9. Cook D, Graeme R, Marshall J, et al. Withdrawal of mechanical ventilation in anticipation of death in the intensive care unit. N Engl J Med 2003; 349:1123-1132.

10. Zib M, Saul P. A pilot audit of the process of end-of-life decision-making in the intensive care unit. Crit Care Resusc 2007; 9:213-218.

11. Schimmer $C$, Gorski $A$, Ozkur M, et al. Policies of withholding and withdrawal of life-sustaining treatment in critically ill patients on cardiac intensive care units in Germany: a national survey. Interact Cardiovasc Thorac Surg 2012; 14:294-299.

12. Thompson BT, Cox PN, Antonelli M, Thijs LJ. Challenges in end-of-life care in the ICU: statement of the 5th International Consensus Conference in Critical Care: Brussels, Belgium, April 2003: executive summary. Crit Care Med 2004; 32:1781-1784.

13. Gristina G, De gaudio R, Mazzon D, Curtis J. End of life care in Italian intensive care units: where are we now? Minerva Anestesiol 2011; 77:911-920.

14. Curtis JR, Engelberg RA. Measuring success of interventions to improve the quality of end-of-life care in the intensive care unit. Crit Care Med 2006; 34 (11 Suppl.):S341-S347.

15. Prendergast $T$, Puntillo K. Withdrawal of life support. Intensive caring at the end of life. JAMA 2002; 288:2732-2740.

16. Quinn JR, Schmitt M, Baggs J, et al. Family members' informal roles in end of life

- decision making in adult intensive care units. Am J Crit Care 2012; 21:43-51.

A study assessing the importance of the role of relatives in the decision-making process in the ICU.

17. Høghaug G, Fagermoen MS, Lerdal A. The visitor's regard of their need for support, comfort, information proximity and assurance in the intensive care unit. Intensive Crit Care Nurs 2011. [Epub ahead of print]

18. Pachman DR, Swetz KM, Mauck WD, et al. An exploratory pilot study of

- palliative medicine compared to anesthesia-pain consultation for pain in patients with cancer. J Support Oncol 2011; 9:113-119.

This study explores the different capacity of anesthesiologists and palliative care

physicians in treating pain and symptoms.

19. Kay S, Husbands E, Antrobus J, Munday D. Provision for advanced pain management techniques in adult palliative care: a national survey of anaesthetic pain specialists. Palliat med 2007; 21:279-284.

20. De Oliveira R, dos Reis $M$, Prado $W$. The effects of early or late neurolytic plexus block on the management of abdominal or pelvic cancer pain. Pain 2004; 110:400-408.

21. Tei $Y$, Morita $T$, Nakaho $T$, et al. Treatment efficacy of neural blockade in specialized palliative care services in Japan: a multicenter audit survey. J Pain Symptom Manage 2008; 36:461-467.

22. Mercadante S, Porzio G, Gebbia V. Spinal analgesia for advanced cancer patients: an update. Crit Rev Oncol Hematol 2011. [Epub ahead of print] 